Accent on communication: The impact of regional and foreign accent on comprehension in adults with aphasia.

\title{
Carolyn Bruce*
}

Language and Communication, Division of Psychology and Language Sciences, University College London

\section{Cinn-Teng To}

Camden Language and Communication Service, 100 Stanhope Street, London NW1 3JX

\section{Caroline Newton}

Developmental Science, Division of Psychology and Language Sciences, University College London

* Corresponding author

Dr C. Bruce, UCL Language \& Communication, Chandler House, 2 Wakefield Street, London, United Kingdom, WC1N 1PF

Tel: +44(0)2076794225

E-mail: c.bruce@ucl.ac.uk 


\section{Introduction}

Increased mobility and migration within and between countries has meant that people around the world are living in ethnically diverse, multicultural communities and more bilingual and multilingual speakers are recipients and providers of services. Increasingly we interact with individuals who speak our language in an unfamiliar regional accent (e.g. English spoken by a native English speaker from a different region) or in a non-native accent (e.g. English spoken by a person whose first language is not English). Previous research indicates that non-native accented speech is less intelligible, takes longer to process and is less accurately understood than speech produced by native speakers, particularly in adverse conditions, such as a noisy environment ${ }^{[1,2]}$. Evidence also suggests that individuals with compromised sensory, cognitive and linguistic skills are even more likely to have difficulties understanding an unfamiliar accent ${ }^{[3]}$. In this paper, we explore the effect that unfamiliar regional and non-native accents have on the comprehension of individuals with aphasia, an acquired language difficulty arising from brain damage.

Every speaker has an accent, a way of pronouncing words that indicates their geographical or social background. Although the production of specific vowels and consonants is a key component of a particular accent, prosody, including rhythmic stress and intonation, also plays a part ${ }^{[4]}$. Deviations from a familiar accent in any of these features can cause difficulties for the listener, although evidence indicates that prosodic differences have a greater effect on comprehension than segmental (i.e. vowels and consonants) differences ${ }^{[5]}$. A variety of factors have been found to affect the comprehension of utterances produced with an unfamiliar accent, including grammatical complexity, familiarity with the topic $^{[6]}$ and with a particular speaker. A speaker's accent may elicit positive or negative reactions in the listener, depending on the stereotypes associated with a particular ethnic group ${ }^{[7]}$, and listeners with negative attitudes towards an accent tend to rate the speaker as more difficult to understand ${ }^{[8]}$. 
In contrast to the extensive research carried out amongst 'healthy' adults, there are still relatively few studies which investigate the processing of unfamiliar native and non-native accents by those with cognitive and communication difficulties. This area of investigation is important as any factor that may act as a communication barrier can have significant implications for the delivery of healthcare services and the long-term rehabilitation of people with acquired communication difficulties. Moreover, difficulties comprehending a non-native accent may limit the participation of individuals with communication difficulties within the community ${ }^{[9,10]}$.

In possibly the first published research on accent processing in this population, Mahendra, Bayles and Tomoeda ${ }^{[11]}$ compared the performance of a group of adults with Alzheimer's disease with a group of elderly individuals on tests of speech discrimination and repetition where stimuli were presented in a familiar American English accent and an unfamiliar Hindi accent. They found that the unfamiliar accent adversely affected the performance of both groups, with significant differences between accent conditions on both tests; a finding mirrored in other more recent research ${ }^{[12,13,14]}$. Although Mahendra et al.'s findings provide valuable insights as to the listeners' difficulties in decoding accented speech, it is not clear how accent affects their ability to comprehend the message.

In contrast to Mahendra et al.’s study, which focused on intelligibility, Burda, Brace and $\operatorname{Hosch}^{[15]}$ assessed the ability of American individuals with and without aphasia to point to written medical sentences that matched utterances produced in English by native and non-native (Mandarin and Spanish) speakers. They found that both groups of participants made a greater number of errors identifying sentences spoken in non-native accents than a familiar native accent. They also found that participants with aphasia were significantly less accurate in their task than those without aphasia. Unfortunately, a number of weaknesses in the design some of which are acknowledged by the authors, including the nature of the stimuli, limit the conclusions that can be drawn from this study. 
More recently, researchers using a sentence-to-picture matching task to investigate comprehension of accented speech, found that a familiar native (English) accent was more accurately understood than an unfamiliar (Nigerian) accent by native British English individuals both with and without aphasia ${ }^{[16]}$. Their control group responded with a higher level of accuracy than adults with aphasia in both accent conditions and there was a significant interaction between accent condition and group, indicating that the adults with aphasia found it significantly more difficult to comprehend sentences in the unfamiliar accent than the control group. Familiarity of accent had no significant impact on response times for either group.

In summary, there is growing evidence that spoken communication between unfamiliar accented (non-native and regional) speakers and native listeners may be problematic, particularly in adverse listening conditions and for those with cognitive and/or communication difficulties. However, it remains to be determined whether some accents are more problematic than others. The present study aimed to investigate whether listeners with aphasia experienced more difficulty with non-native rather than regionally-accented English. In order to do this, we used a spoken sentence verification task which includes sentences that are all obviously true or false. The task provides a measure of both the speed and accuracy of auditory input processing, and we hypothesised that responses would be slower and less accurate in the foreign accent condition.

\section{Methods}

Ethical approval for this project was gained from a Departmental Ethics Committee.

Participants. Listeners for the sentence verification task were 17 adults with aphasia (seven female; ten male) between the ages of 38 and 79 with a mean age of 61.88 years and 17 adult control participants (eleven female; six male) between the ages of 37 and 76 with a mean age of 54.06 years. An 
independent samples t-test revealed that there was no significant difference in age between the two groups $t(32)=1.93, p=0.06$. Participants were recruited consecutively through posters and by word of mouth. All participants were resident in the South East of England, and all but one spoke English as their first language. This individual's first language was Serbian but she had lived in England and spoken English as her primary language for more than 35 years. None of the participants reported any regular contact with the unfamiliar regional or non-native accents used in the study, though they were familiar with the SSBE accent. Participants with aphasia had language difficulties caused by dominant hemisphere stroke. All were at least six months post-onset and attended a community clinic for acquired communication disorders in South East England. They presented with a range of communication difficulties and levels of severity (as measured by the Western Aphasia Battery $\left.(\mathrm{WAB})^{[17]}\right)$. Eligibility criteria also included good levels of pre-morbid English; average pure tone thresholds (at 500, 1000, $2000 \mathrm{~Hz}$ ) at or below $25 \mathrm{~dB}$ HL in at least one ear and a score at or above $85 \%$ on the "Speech Discrimination Screening Task" of the Arizona Battery for Communication Disorders of Dementia ${ }^{[18]}$ indicating adequate speech discrimination. The 17 participants without aphasia were adults with no history of neurological deficit and no hearing difficulties. Demographic data for the participants with aphasia are provided in table 1.

Table 1: Demographic data for the participants with aphasia.

\begin{tabular}{|c|c|c|c|c|}
\hline $\begin{array}{l}\text { Participants } \\
\text { with aphasia }\end{array}$ & Age & Gender & $\begin{array}{l}\text { Years post } \\
\text { stroke }\end{array}$ & $\begin{array}{l}\text { WAB classification (aphasia quotient; auditory } \\
\text { comprehension component score) }\end{array}$ \\
\hline 1 & 38 & $\mathrm{~F}$ & 7 & Anomic $(88.6 ; 9.3)$ \\
\hline 2 & 46 & $\mathrm{~F}$ & 2 & Anomic $(74.8 ; 8.7)$ \\
\hline
\end{tabular}




\begin{tabular}{|c|c|c|c|c|}
\hline 3 & 56 & $\mathrm{M}$ & 6 & Anomic $(74.4 ; 8.3)$ \\
\hline 4 & 59 & $\mathrm{M}$ & 2 & Anomic $(73.5 ; 7.85)$ \\
\hline 5 & 60 & $\mathrm{~F}$ & 2 & Anomic $(93.2 ; 9.6)$ \\
\hline 6 & 63 & $\mathrm{~F}$ & 5 & Anomic $(91.6 ; 10)$ \\
\hline 7 & 75 & $\mathrm{M}$ & 12 & Anomic $(92.8 ; 9.8)$ \\
\hline 8 & 77 & $\mathrm{~F}$ & 17 & Anomic $(98 ; 10)$ \\
\hline 9 & 52 & $\mathrm{M}$ & 3 & Conduction $(84.4 ; 10)$ \\
\hline 10 & 64 & $\mathrm{M}$ & 1 & Conduction $(74.7 ; 8.85)$ \\
\hline 11 & 64 & $\mathrm{M}$ & 17 & Conduction $(65.6 ; 8.5)$ \\
\hline 12 & 72 & $\mathrm{~F}$ & 4 & Conduction $(56.6 ; 8.2)$ \\
\hline 13 & 74 & $\mathrm{M}$ & 7 & Conduction $(71.4 ; 7.7)$ \\
\hline 14 & 75 & $\mathrm{M}$ & 1 & Conduction $(81.3 ; 9.95)$ \\
\hline 15 & 79 & $\mathrm{~F}$ & 1 & Conduction $(66.4 ; 7.6)$ \\
\hline 16 & 50 & $\mathrm{M}$ & 1 & Broca's $(54.6 ; 9.4)$ \\
\hline 17 & 48 & $\mathrm{M}$ & 4 & Wernicke's $(62.3 ; 6.45)$ \\
\hline
\end{tabular}


Accents. Accent samples were provided by three speakers (average age 40 years), who were all female. The accents were selected as representative of three accents that differ from each other in segmental and/or prosodic features:

- a familiar Southern Standard British English (SSBE) accent. The speaker was born and raised in London and had spent all her life in the area. As with other native accents of English, her natural rhythmic pattern was stress-timed.

- an unfamiliar native accent from the lower north Humberside region in the North East of England (Grimsby) - differing from the familiar accent by segmental features only (for example, 'Luke' and 'look' are pronounced identically). The speaker was born and lived in Grimsby before moving to South East England, where she had been for nearly nine years. The rhythmic-prosodic patterns of her speech were also stress-timed.

- an unfamiliar non-native Chinese accent - differing from the familiar standard accent both in segmental pronunciation and prosodic-rhythmic qualities. The speaker was born in Hong Kong and lived there for 21 years before moving to England, where she had lived for 29 years. She was a native speaker of Cantonese and learned English when she moved to England. Her production of English, though fluent, is influenced by both segmental features (for example, 'bet' and 'bat' are pronounced identically) and the syllable-timed prosodic pattern of her first language.

\section{Materials}

A simple sentence verification task based on everyday knowledge, with minimal visual, cognitive and physical dexterity demands, was selected so the effect of accent on comprehension was clear. Sixty stimuli sentences were constructed specifically for this study, rather than using published materials (e.g. The Speed and Capacity of Language Processing (SCOLP) Test ${ }^{[19]}$ ) whose stimuli were judged 
too demanding linguistically for some of the participants with aphasia. The sentences were single subject-verb-object (SVO) clauses consisting of three words, for example, 'Elephants have trunks' and 'Sharks are flowers'. There were an equal number of true and false sentences, which were randomly divided between the three different accent conditions (familiar - unfamiliar native - non-native), giving two practice sentences and 20 test sentences, 10 true and 10 false, in each condition. An equal number of noun categories (e.g. animals, fruit) and sentence lengths (in syllables) were used in each accent.

\section{Procedure}

Recording speech samples. Speakers were recorded in a quiet but not sound-proofed room using a Creative Zen Micro mp3 recorder. Prior to recording, the speakers read the sentences and practised them aloud if they felt it was necessary. Recordings of each stimulus sentence for each speaker were separated into individual sound files using Magix Audio Cleaning Lab.

Experimental task. All listeners were tested individually in a quiet but not sound-proofed room. A Dell Optiplex SX280 desktop computer with 17" monitor, equipped with a bespoke computer programme written in Visual Basic .NET, presented the stimuli and recorded response time and accuracy data for the sentence verification task. The listeners sat directly in front of the monitor, and visual stimuli, a box containing a tick for 'yes' and a box containing a cross for 'no', were displayed centrally on the monitor six inches apart. The auditory stimuli were played via the PC's internal speakers. Listeners were given a choice of using either a touch-screen or a mouse to respond to each stimulus. Once they had selected a response mode, the same method of response was used for all the stimuli. The listeners placed the mouse or the hand they were using to respond on a centrally located marker a fixed distance from the computer and were asked to return to this position between each trial. The auditory stimuli for each accent condition were played in turn and the listeners were asked to indicate whether the sentence 
was true or not by pressing either the tick or the cross icon before moving on to the next item. The listeners were instructed to respond to each sentence as quickly and as accurately as possible. Stimulus presentation was controlled by the tester. Participants were given two practice items before each accent condition (with a total of six practice items) in order to familiarise them with the task procedures and to expose each accent equally, regardless of which accent condition was presented as the first condition. Comprehension accuracy (the number of correct 'yes' and 'no' responses in each condition) and response times were determined for each sentence in each condition. Response time was calculated from the end of each sentence to when the icon on the screen was pressed, and was averaged for the correct responses only.

\section{Results:}

Statistical analysis of the data was carried out with comprehension accuracy and response time as the dependent variables.

Comprehension accuracy: A two-way mixed ANOVA 2x3 was carried out with accent condition as the within subjects factor with three levels (Standard-Regional-Non-native) and group condition as the between-subjects factor (participants with and without aphasia). One outlier (participant 11) with scores three standard deviations below the group mean in the Standard condition was excluded from analysis. The analysis showed a highly significant main effect of accent $(F(2,30)=26.465, \mathrm{df}=1.487$, $p<0.001)$ and group $(F(2,30)=43.835, \mathrm{df}=1, p<0.001)$, and a significant interaction effect $(F(2,30)=3.872, \mathrm{df}=1.487, p=0.04)$. 
Interaction effects were investigated using a follow-up ANOVA approach (see table 2 for mean scores). Visual inspection of the data indicates that the aphasic group were less accurate in all three accent conditions compared to the control group.

Table 2: Mean accuracy scores (out of 20) and standard deviations (in brackets) made by the participants with aphasia (outlier excluded) and participants without aphasia across the three accent conditions.

\begin{tabular}{|l|c|c|c|}
\hline & Standard accent & Regional accent & Non-native accent \\
\hline Participants with aphasia & $18.1(1.6)$ & $17.4(1.2)$ & $15(2.5)$ \\
\hline Participants without aphasia & $19.7(0.4)$ & $19.5(0.6)$ & $18.4(1.2)$ \\
\hline
\end{tabular}

A Bonferroni-corrected t-test indicated that both the aphasic group and the control group were significantly less accurate with the non-native accent compared to the standard (aphasics: $p=0.001$, controls: $p=0.001$ ) and regional (aphasics: $p=0.009$, controls: $p=0.021$ ) accents. However, there were no significant differences in scores between the standard and regional accent for either group. An independent samples t-test, comparing the means for each accent condition, showed that the aphasic group made significantly more errors in the standard $(t(31)=3.916, p<0.001)$, regional $(t(31)=4.063$, $p<0.001)$ and non-native $(t(31)=5.492, p<0.001)$ accent conditions compared to the control group. However, accuracy scores for the control group are subject to ceiling effects, reflecting the simplicity of the task. 
Response time: Once again, true outliers (two listeners from the group of participants with aphasia: $3 ; 11)$ were identified in the data and excluded from the analysis. Another two-way mixed ANOVA 2x3 was conducted, with the accent condition as the within-subjects factor with three levels (standard-regional-non-native) and the group as the between-subjects factor with two levels (aphasics and controls). A main effect of group was found to be highly significant $(F(2,30)=22.837, p<0.001)$, where the participants with aphasia responded more slowly than the control group, and a significant effect of accent condition $(F(2,30)=4.352, p=0.02)$. However, interaction effect between accent and group was not significant $(F(2,30)=2.102, p=0.131)$.

Paired samples t-tests showed no significant differences between accent conditions for the group of participants with aphasia. Paired samples t-tests comparing the means for each accent condition for the control group showed that they responded significantly more slowly in the non-native accent $(M=1262 \mathrm{~ms}, \mathrm{SD}=526.17)$ compared to the standard $(\mathrm{M}=923, \mathrm{SD}=357.90)(t(16)=8.257$, $p<0.001)$ and regional accent $(t(16)=4.474, p<0.001)$. However, there was no significant difference in mean response times between the standard and regional accent $(\mathrm{M}=962, \mathrm{SD}=249.71)(t(16)=-1.410$, $p=0.178)$ (see figure 1$)$.

Figure 1: Mean response times for group by accent condition (with outliers excluded).

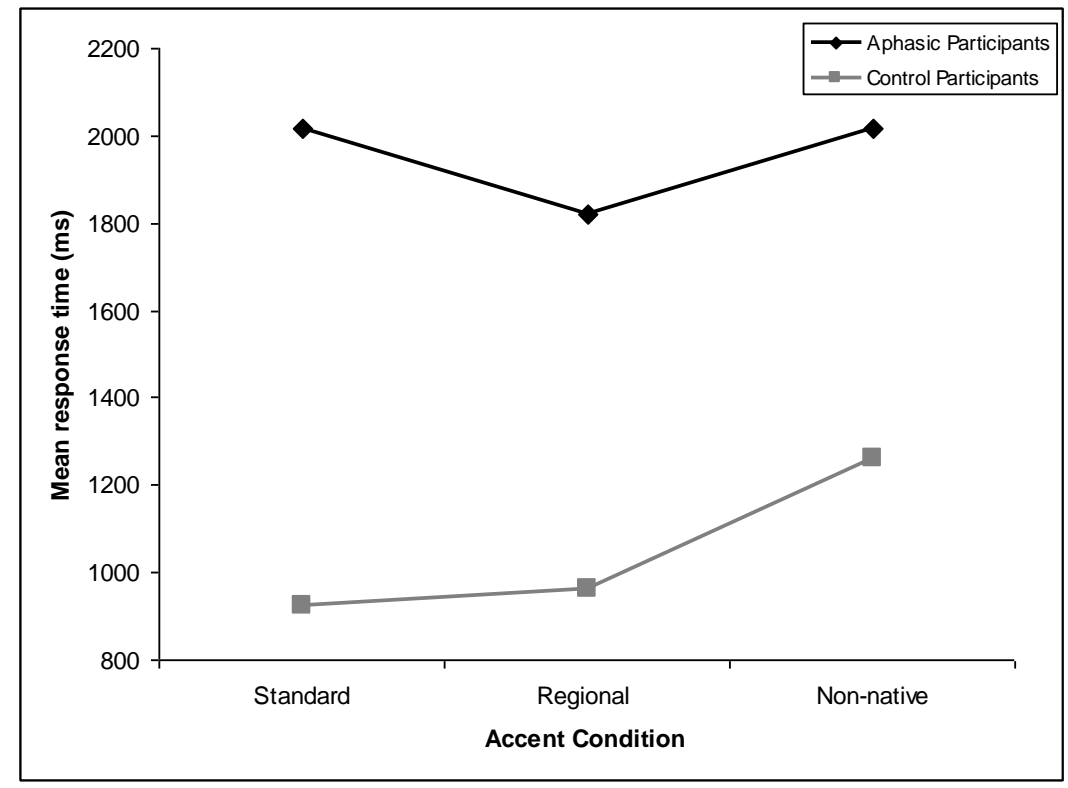




\section{Discussion:}

This study investigated whether an unfamiliar non-native accent, which differs in both segmental and prosodic factors to a familiar native accent, had a stronger impact on comprehension of simple sentences in adults with aphasia than an unfamiliar regional accent. Comprehension was established by accuracy of yes/no responses to stimuli and processing time was estimated using response times calculated from the end of the sentences. Data was collected from individuals with and without aphasia.

Over both groups comprehension was poorer when listening to the unfamiliar regional accented and unfamiliar non-native accented utterances than the familiar accented utterances. Moreover, the non-native accent was significantly more difficult to comprehend than the other two accents and this difficulty was significantly more marked for the listeners with aphasia. These results suggest that an unfamiliar non-native accent, which deviates in both segmental and prosodic characteristics from a familiar accent, has a greater adverse effect on sentence comprehension than an accent that differs by segmental features alone, though the experimental design used here does not allow us to unpick this further. These results support findings that an unfamiliar non-native accent reduces accuracy of sentence comprehension in native listeners without aphasia ${ }^{[20,13]}$ and individuals with aphasia ${ }^{[16]}$.

For response time, the participants with aphasia were much slower overall than the participants without aphasia, but the interaction found with the accuracy scores was not mirrored here. The findings indicate that the participants without aphasia responded significantly more slowly to the unfamiliar Chinese accent than to the familiar standard and unfamiliar regional accent. This supports findings from published studies, which suggest that an unfamiliar accent requires more effortful processing and increases the response times for listeners ${ }^{[2,21]}$. Although the expected pattern of response times was found for the participants without aphasia, individuals with aphasia presented with response times that were surprising. Overall they showed only $1 \mathrm{~ms}$ difference in response time between the familiar standard accent and the unfamiliar Chinese accent and responded most quickly to the unfamiliar 
regional accent. A possible explanation for this finding may be that their general slow response rates have masked effects of accent. Slow responses may reflect their caution at responding before they have processed all the information presented.

One participant with aphasia (participant 11) appears as an outlier in terms of both accuracy and speed of response: performing at a much lower level than other participants in that group. He reported an inability to hold sentences in short-term memory which may have played a part. It may also be that there were specific aspects of the familiar accented speaker which were particularly problematic for this individual, but which we are unable to unpick within the scope of this study.

Overall, these findings raise questions which could be addressed in future research. For example, pragmatic decisions on participant recruitment meant that our group of individuals with aphasia contained those with a variety of aphasia types and ranges of severity, so that it is difficult here to unpick what effect type or severity of aphasia may have on accent processing. In addition, the task used in this study was very simple; different results may be obtained with more demanding tasks (e.g. a comprehension task involving sentences with a variety of linguistic structures), or indeed outside of an experimental setting.

The results of this investigation have important clinical implications for improving communication between non-native accented speakers and native listeners. There is much that is positive about globalisation. However, we need to recognise that for some people there may be a downside. The evidence suggests that even when both parties know and are proficient users of the English language they may not always understand one another, particularly if their accents differ in segmental and prosodic properties. Even in this simple yes/no judgement task, accent was found to have a marked effect on performance. It is likely that when the listening conditions are poor or when the task demands are linguistically and cognitively greater (for example when the spoken utterance consists of longer and more complex sentences or the response required from the listener involves more 
extensive output or when communication takes place in a noisy environment) then accent will exert an even stronger effect on performance. Moreover, in this study we were only investigating the effect of accent on performance: it was simply the manner of pronunciation that was different between speakers. In many cases, individuals from other regions will speak a dialect, which encompasses grammar and vocabulary together with the pronunciation variation referred to in this paper as 'accent' ${ }^{\text {'22] }}$.

These findings suggest that health professionals working in rehabilitation may need to take more care in conversations with patients with accents different to their own. For example, they will need to ensure that the patient has understood what has been said and, when this is not the case, supplement their speech with information in a different medium. This is particularly important when assessing individuals with aphasia, as basing conclusions on a single interaction where speakers have different language or regional backgrounds may lead to underestimations of that person's communicative abilities. Observing patients interacting with a broader range of people would be one solution. Speakers with a non-native accent, but particularly one that is perceived to be 'strong', should consider the effect this might have on their comprehensibility and should at least take steps to minimise other variables that might compromise comprehension, such as speed of delivery, volume, background noise and poor visibility. This will ensure that assessment of functioning reflects the individual's ability and that rehabilitation is targeted appropriately and achieves its full potential. 


\section{Acknowledgements}

The authors wish to thank the participants and speakers who contributed to the study. 


\section{Declaration of interest}

The authors report no conflicts of interest. The authors alone are responsible for the content and writing of the paper. 


\section{References}

[1] Rogers CL, Dalby J, Nishi K. Effects of noise and proficiency level on intelligibility of Chineseaccented English. Language and Speech 2004;47:139-54.

[2] Munro M, Derwing TM. Processing time, accent, and comprehensibility in the perception of native and foreign-accented speech. Language and Speech 1995;38:289-306.

[3] Wingfield A, Tun PA, McCoy SL. Hearing loss in older adulthood: What it is and how it interacts with cognitive performance. Current Directions in Psychological Science 2005;14:144-8.

[4] Wells JC. Accents of English. Cambridge: Cambridge University Press 1982.

[5] Anderson-Hsieh J, Koehler K. The effect of foreign accent and speaking rate on native speaker comprehension. Language Learning 1988;38:561-613.

[6] Gass S, Varonis EM. The effect of familiarity on the comprehensibility of non-native speech. Language Learning 1984;34:65-89.

[7] Lippi-Green R. English with an Accent. London and New York: Routledge 1997

[8] Eisenstein M, Verdi G. The intelligibility of social dialects for working-class adult learners of English. Language Learning 1985;35:287-98.

[9] Howe TJ, Worrall LE, Hickson LM. Interviews with people with aphasia: Environmental factors that influence their community participation, Aphasiology 2007;22:1092-120.

[10] Le Dorze G, Brassard C, Larfeuil C, Allaire J. Auditory comprehension problems in aphasia from the perspective of aphasic persons and their families and friends, Disability and Rehabilitation 1996; $18 ; 550-8$.

[11] Mahendra N, Bayles KA, Tomoeda CK. Effect of an unfamiliar accent on the repetition ability of normal elders and individuals with Alzheimer's Disease. Journal of Medical Speech-Language Pathology 1999;7:223-30. 
[12] Adank P, Janse E. Comprehension of a novel accent by young and older listeners. Psychology and Aging 2010;25:736-40.

[13] Ferguson SH, Jongman A, Sereno JA, Keum KA. Intelligibility of Foreign-Accented Speech for Older Adults with and without Hearing Loss. Journal of the American Academy of Audiology 2010;21:153-62.

[14] Burda AN, Hageman CF. Perception of accented speech by residents in assisted-living facilities. Journal of Medical Speech-Language Pathology 2005;13: 7-14.

[15] Burda AN, Brace A, Hosch A. Aphasia and accent in identifying medical sentences. Perceptual and Motor Skills 2007;104:1375-8.

[16] Dunton J, Bruce C, Newton C. Investigating the impact of unfamiliar speaker accent on auditory comprehension in adults with aphasia. International Journal of Language \& Communication Disorders 2011;46:63-73.

[17] Kertesz A. Western aphasia battery (WAB). (San Antonio: The Psychological Corporation) 1982. [18] Bayles KA, Tomoeda CK. The Arizona Battery for Communication Disorders of Dementia. Tucson, AR: Canyonlands Publishing 1991

[19] Baddeley AD, Emslie H, Nimmo-Smith I. The Speed and Capacity of Language Processing (SCOLP) Test. Bury St Edmunds, UK: Thames Valley Test Co 1992.

[20] Adank P, Evans B, Stuart-Smith J, Scott SK. Comprehension of familiar and unfamiliar native accents under adverse listening conditions. Journal of Experimental Psychology: Human Perception and Performance 2009;35:520-9

[21] Clarke CM, Garrett MF. Rapid adaptation to foreign-accented English. Journal of the Acoustical Society of America 2004;116:3647-58.

[22] Hughes A, Trudgill P, Watt D. English accents and dialects: an introduction to social and regional varieties of English in the British Isles. Fourth Edition. London: Hodder Arnold 2005 